\title{
Calcium and vitamin D supplementation in fragility fractures: are we up-to-date?
}

\author{
C. Tsang and J. Youngman \\ Department of Trauma and Orthopaedics, University College Hospital, London NWI 2BU, UK
}

300,000 cases of fragility fracture occur each year in the UK costing $£ 2$ billion in medical and social care ${ }^{(1)}$. Current projections indicate that the number of hip fractures is likely to increase, doubling by $2050^{(1)}$. The Cochrane Systematic review concluded that combined calcium and vitamin D supplementation significantly reduces the incidence of fractures in the elderly population ${ }^{(2)}$. Guidance from the National Institute for Health and Clinical Excellence and the British Orthopaedic Association regarding secondary prevention of osteoporosis recommends that supplementation should be prescribed if clinicians cannot be confident that calcium and vitamin D levels are replete $^{(1,3)}$. Prevalence of vitamin D deficiency is reported to be up to $70 \%$ in patients who fall ${ }^{(4)}$. This audit aims to evaluate the use of calcium and vitamin D supplementation in patients with fragility fractures.

The study period was over five months between January and May 2011. All patients who presented with fragility fractures with an admission episode were included in the study. The rate of prescription of calcium and vitamin D supplements upon discharge was evaluated.

67 cases were assessed. Mean age was 73 years (range 53-99). 69\% were female. Presenting complaints were femoral fractures (58\%), fractures of distal radius (18\%), humerus (10\%), ankle (8\%), pelvis (3\%) and tibia (3\%). 9 cases (13\%) had a history of falls, of which 5 had sustained a previous fracture. $15 \%$ of all patients had a diagnosis of osteoporosis.

In 30 out of 67 cases (45\%), calcium and vitamin D supplementation were prescribed upon discharge. Bone density assessments and bone protection medications were considered in 22 cases, as documented in discharge summary. Prescription rate within this group was $50 \%$.

This audit has shown that the majority of patients admitted with fragility fractures did not receive a prescription of calcium and vitamin D supplementation on discharge. This accounted for $55 \%$ of cases. It was noted that considerations for bone health assessments or intervention did not improve supplementation rate. There is an obvious margin for improvement. This carries both financial and clinical implications. Following the introduction of the Best Practice Tariff in April 2010, which encourages the use of bone protection medications, failure to comply could lead to a loss of $£ 445$ per case ${ }^{(5)}$. Furthermore, osteoporosis failed to be incorporated into the Quality and Outcomes Framework of the revised GP contract ${ }^{(3)}$. Prescription of supplementations on discharge can act to raise awareness of bone health and nutrition amongst primary care physicians in this vulnerable patient group. Targets for intervention includes regular education of junior members of the orthopaedic team bearing in mind that regular turnover of staff may contribute towards inconsistency of care delivered. This audit aims to raise awareness of the importance of calcium and vitamin D supplementation in patients with fragility fractures to both primary and secondary care clinicians at a time where there appears to be significant variations in clinical practice.

1. The care of patients with fragility fractures (2007) British Orthopaedic Association.

2. Avenell A, Gillespie WJ \& Gillespie LD et al. (2009) Cochrane Database of Systematic Reviews.

3. Technology appraisal guidance 161 (2008) NICE.

4. Annweiler C, Montero-Odasso M, Schott AM et al. (2010) J Neuroeng Rehabil 7:50.

5. Wilson H, Harding K \& Sahota O (2010) National Hip Fracture Database Resources. 